\title{
Adaptive Noise Image Enhancement Method Based on Genetic Algorithm in Nonsubsampled Contourlet Domain
}

\author{
Yang Li, Shengyong Chen, Wei Huang \\ TianJin University of Technology \\ TianJin, 300384 \\ China \\ liyang_19901222@163.com
}

\begin{abstract}
In order to suppress the noise adaptively while enhancing the image details, an adaptive image enhancement method based on genetic algorithm in Nonsubsampled Contourlet (NSCT) domain is proposed. The optimal parameters of the image enhancement function are obtained adaptively through genetic algorithm (GA). The NSCT high frequency sub-bands coefficients are processed by the image enhancement function, the coefficients which are less than the low threshold are set to 0 and the coefficients between the low threshold and the high threshold are enhanced. Experimental results demonstrate that the proposed method can adaptively enhance the image details and suppress noise at the same time, the detail variance is significantly improved, and a better visual effect is obtained. The results processed by the proposed method are better than that by classical enhancement methods.
\end{abstract}

Keywords: Image Enhancement, NSCT, Noise, Adaptive, GA

Received: 9 April 2019, Revised 5 August 2019, Accepted 24 August 2019

DOI: $10.6025 / \mathrm{jmpt} / 2019 / 10 / 4 / 138-151$

(C) 2019 DLINE. All Rights Reserved

\section{Introduction}

The quality of images is affected by the imaging environment, imaging equipment and transmission system in the process of acquisition and transmission. So usually the final images contain noise and it is difficult to identify the details. The purpose of image enhancement is to improve the image quality and enhance particular target information according to the follow-up image processing requirements, and suppress the noise at the same time. Recently, the image enhancement research focuses on enhancing the edges or texture details of image, rather than improving the dark region of image [1].

The traditional spatial image enhancement methods usually include: histogram equalization (HE) [2], contrast limited adaptive histogram equalization (CLAHE) [2], guided filter [3], gray level transformation [4] and so on. Histogram equalization can effectively improve the overall contrast of the image and is applicable to the image which has a narrow dynamic range of gray. This method is easy to understand and has been used widely. But it also has limitations [5]. The pixels with different gray levels are merged into the same gray level, so the details of the image are lost. Moreover, the algorithms based on histogram equalization would produce an unsatisfactory outcome, such as amplifying background noise. Guided filter is a kind of local enhancement filter, which has the characteristic of keeping edges. This method runs fast and works well in the noise free images [6]. If there is 
noise in the image, the noise will be enlarged. Then the image details are lost. Gray level transformation is a pixel value mapping process, such as the piecewise linear transformation, which is easy to understand and runs fast. But the parameters of gray mapping function need to be set manually [7]. The method based on Fourier transform will amplify the noise while enhancing the contrast of the image, which also would make the image details lost. The bilateral filter [8] is a non-linear, edge-preserving and noise-reducing smoothing filter for images. The intensity value at each pixel in an image is replaced by a weighted average of intensity values from nearby pixels. Its processing objects are noised images and it works well in suppressing noise while enhancing image details.

As the multi-scale analysis is introduced, the image enhancement method based on wavelet has obtained good results $[9,10]$. The method based on the wavelet transform outperforms in the details enhancement and noise suppression. But the wavelet basis function is anisotropic, which can not capture image edge details effectively. However, most of the image information is contained in the image edges.

With the continuous development of multi-scale geometric analysis research in these years, different new methods based on new multiscale transforms have been proposed, such as the ridgelet, curvelet, and contourlet transform [11] etc. The contourlet transform is proposed to address the lack of geometrical structure in the separable two-dimensional wavelet transform. The research results show that the performance of the image enhancement and image denoising based on the new multiscale transforms are better than those based on the wavelet transform. The contourlet transform is a real 2-D image representation using a directional filter and a laplacian pyramid, which can effectively capture contours in an image and can achieve better expression of image than the wavelet transform. It also has the anisotropic characteristics. Because of the process of downsampling and upsampling, the contourlet transform is shift-variance and always has Gibbs phenomena around singularities. The NSCT [12] is proposed by Cunha. The NSCT has better performance in image denoising and enhancement applications. The NSCT is composed by the nonsubsampled pyramid filter and the nonsubsampled direction filter, so it is shift-invariance. The traditional methods of image enhancement based on transform domain set the parameter manually [13-15]. So it is too casual and subjective. In order to get a good result, the parameters are very important and need to be set many times.

A novel adaptive image enhancement method based on genetic algorithm in nonsubsampled Contourlet domain is proposed in this paper. We propose an improved image enhancement function and an image evaluation method. The image enhancement function is used to nonlinearly shrink and stretch the NSCT coefficients. The proposed image evaluation function is used to evaluate the fitness of individuals in genetic algorithm (GA). The optimal parameters of image enhancement function are obtained by GA. Experimental results show that the proposed method in this paper can effectively suppress the noise while enhancing the images details adaptively.

\section{Nonsubsampled Contourlet Transform}

The nonsubsampled Contourlet Transform is also a real 2-D image representation [12]. The NSCT has better directional frequency localization and a fast implementation. The NSCT consists of the nonsubsampled pyramid filters banks (NSPFB) and the nonsubsampled direction filter banks (NSDFB). The two independent parts are shift-invariant, so the NSCT has the shiftinvariant performance. The NSPFB is a shift-invariant filtering structure. The reconstruction condition [16] is shown as:

$$
\begin{gathered}
H_{0}(Z) G_{0}(Z)+H_{1}(Z) G_{1}(Z)=1 \\
H_{0}(Z)+H_{1}(Z)=1 \\
G_{0}(Z)+G_{1}(Z)=1
\end{gathered}
$$

Where, $H_{0}(Z)$ is a low pass decomposition filter, $H_{1}(Z)$ is a high pass decomposition filter, $G_{0}(Z)$ is a low pass reconstruction filter, and $G_{1}(Z)$ is a high pass reconstruction filter.

The DFB is not shift-invariant. A shift-invariant directional expansion is obtained with a nonsubsampled DFB (NSDFB).

The NSDFB is constructed by eliminating the downsamplers and upsamplers. All filters are upsampled by a quincunx matrix given

Journal of Multimedia Processing and Technologies Volume 10 Number 4 December 2019 
as:

$Q=\left[\begin{array}{rr}1 & 1 \\ 1 & -1\end{array}\right]$

NSDFB uses singular points of the same direction to synthesize the NSCT coefficients. The multiscale property of the NSCT is obtained from a shift-invariant filtering structure. Any directions can be decomposed in every scale sub-bands, and the number of directions usually is $2 n(n \in N)$.

Figure 1 displays a high level view of the proposed NSCT. The result is a tree-structured filter bank that splits the frequency plane in the directional wedges shown in Figure 2.

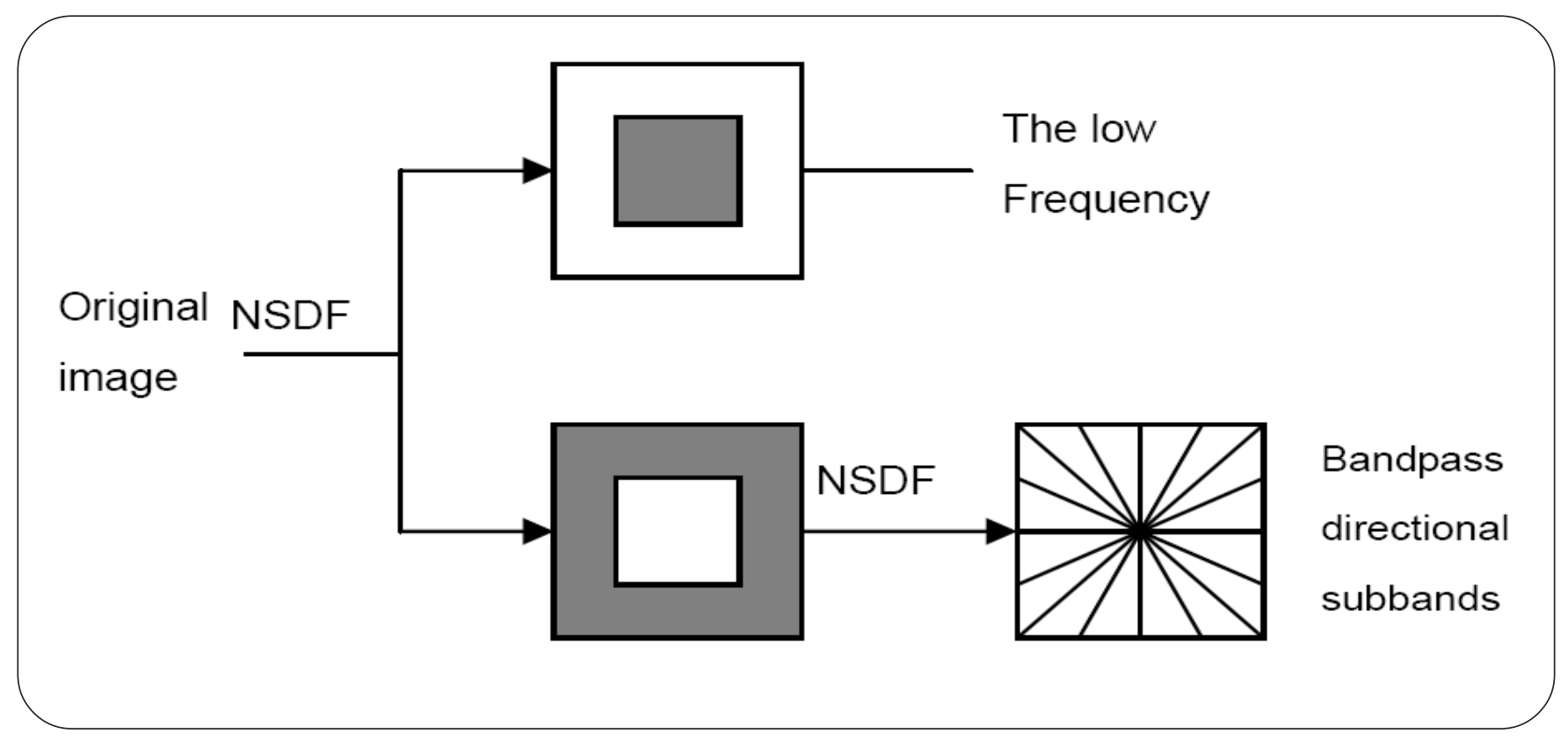

Figure 1. Nonsubsampled filter bank structure

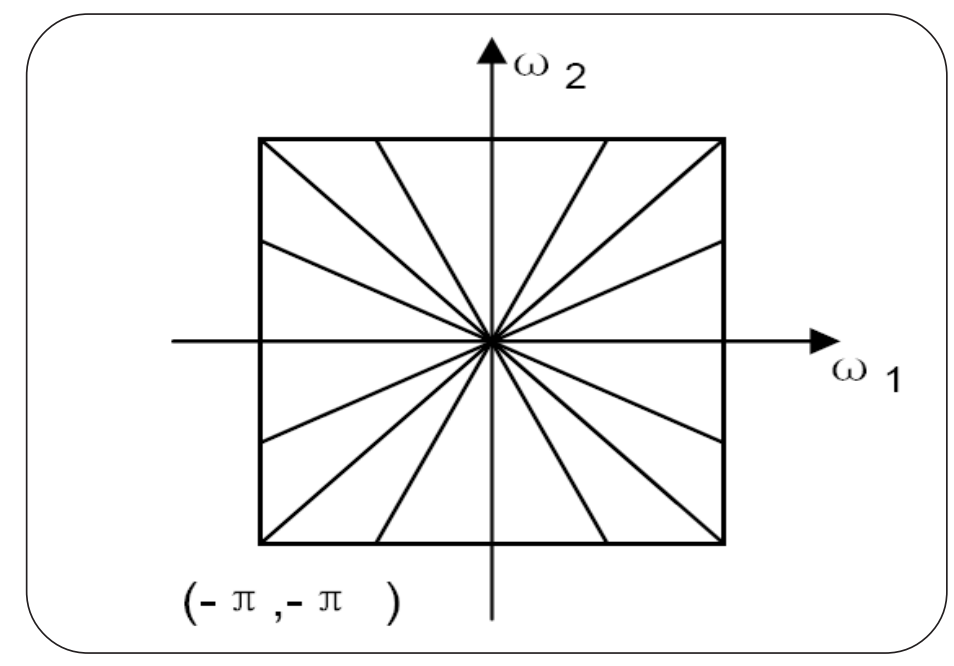

Figure 2. The frequency decomposition

The NSCT can effectively use a few coefficients to capture the edges and contours in the image. And the edges and contours are the main features of the natural images. The image is composed into high frequency directional sub-bands and low frequency 
sub-band by NSCT. The main energy of the image is concentrated in the low frequency sub-band. The edge details and noise are concentrated in the high frequency directional sub-bands. Because the NSCT has the de-correlation performance, the energy of edge coefficients is concentrated and the amplitude value of edge coefficient is big. And the energy of noise coefficient is dispersed and the amplitude value is small. The NSCT can also effectively capture contours in an image. For example, decomposition level is 3 . The third decomposition level is shown in figure 3 . There are four directions in level 3. From figure 3 , we can clearly see that the NSCT can effectively capture directional information. Since the NSCT is good at representing images with edges, it is a good way for edge enhancement.

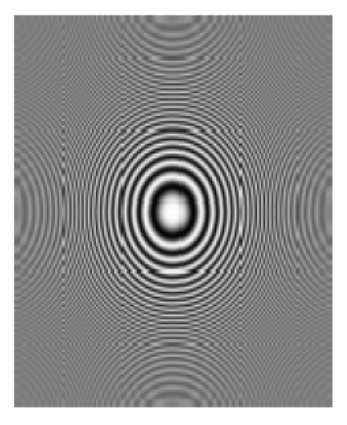

(1) Original image

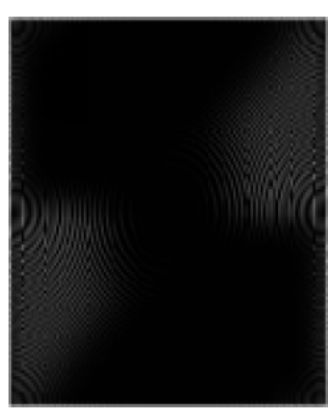

(2) Direction 1

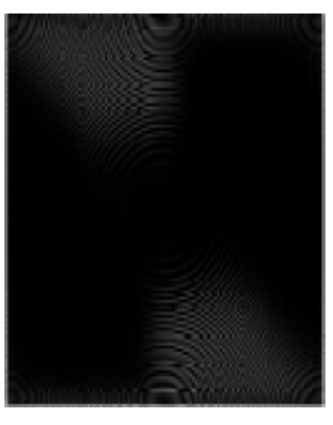

(3) Direction 2

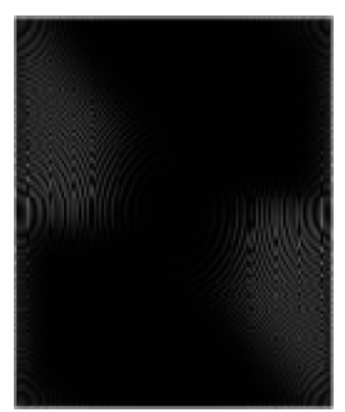

(4) Direction 3

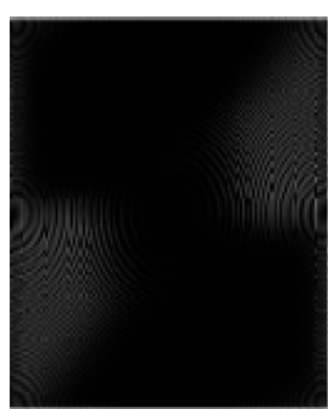

(5) Direction 4

Figure 3. Decomposition by NSCT

\section{Image Enhancement in Nonsubsampled Contourlet Domain}

\subsection{Improved Nonlinear Image Enhancement Function}

A contrast enhancement method is proposed by Starck et al. in the curvelet transform [17]. In order to enhance the image details, they proposed an enhancement function which is given as:

$$
y=\left\{\begin{array}{llc}
1 & \text { if } & 0 \leq x<c \sigma \\
\frac{x-c \sigma}{c \sigma}\left(\frac{m}{2 c \sigma}\right)^{p}+\frac{2 c \sigma-x}{c \sigma} & \text { if } & c \sigma \leq x \leq 2 c \sigma \\
\left(\frac{m}{x}\right)^{p} & \text { if } & 2 c \sigma<x \leq m \\
\left(\frac{m}{x}\right)^{S} & \text { if } & m<x \leq 1
\end{array}\right.
$$

Here, $\sigma$ is the noise standard variance, $m$ is the value under which coefficients are amplified, $p$ determines the degree of nonlinearity and $s$ introduces dynamic range compression. $c$ is a normalization parameter, and $c$ which are more than 3 guaranties that the noise will not be amplified. This enhancement function does well in enhancing image details [17].

However, this function does not effectively suppress the noise.

In order to enhance the image details and suppress the noise, we proposed an image enhancement function. The NSCT coefficients can be modified in order to enhance edges in an image. The high frequency coefficients of the image can be divided into strong edges, weak edges and noise. In order to enhance weak edges and suppress noise, a nonlinear mapping function is proposed in this paper. The proposed enhancement function is shown in equation (6). Where $x$ are the normalized high-frequency sub-bands coefficients. The $m 1$ and $m 2$ respectively represent low threshold and high threshold. $y$ represents the processed coefficient. sign is used to get the symbols of $x$. 


$$
y= \begin{cases}0 & \text { if } \quad 0 \leq|x|<m 1 \\ \operatorname{sign}(x)\left\{m 1+(m 2-m 1)\left(\frac{|x|-m 1}{m 2-m 1}\right)^{a^{b}}\right\} & \text { if } \quad m 1<|x|<m 2 \\ \operatorname{sign}(x)|x| & \text { if } \quad m 2 \leq|x| \leq 1\end{cases}
$$

From equation (6), we can see clearly that the improved function differs from the Starck's enhancement function. The absolute value of coefficients which are less than $m 1$ are attributed to noise and are set to zero in our enhancement function. And the absolute values of coefficients which are more than $m 2$ keep unchanged. If $x$ is less than $m 1, x$ is classified as noise and is set to 0 . If $x$ is larger than $m 1$ and less than $m 2, x$ is classified as the weak edges and is enhanced by the nonlinear mapping function. If $x$ is larger than $m 2$, it is classified as strong edges and is retained.

\subsection{Image enhancement evaluation function}

In order to get the optimal parameters $(m 1, m 2, a, b)$ adaptively, an image enhancement evaluation function is needed. A high quality image should have little noise and clear details, so the DV(detail variance)/BV(background variance) [18], SNR (Signal to Noise Ratio) and canny algorithm are adopted in the proposed image enhancement evaluation function.

The DV/BV evaluation method is to divide the image into detail pixels and background pixels. A sliding window is used to calculate the local variance of each pixel. The $3 * 3$ window is adopted in this paper, and the threshold T is got in literature [19]. If the pixel variance is less than the threshold $\mathrm{T}$, then it is classified as the background pixel. Else the pixel is classified as the detail pixel. The BV is the mean of all the background pixel variance, and the DV is the mean of all detail pixel variance. Usually if the image details are not clear in image, the DV/BV value is relatively little and if the image details are clear in image, the DV/BV value is relatively large. If there is noise in the image, the $\mathrm{DV} / \mathrm{BV}$ value is relatively large, but the image details are vague.

The canny algorithm is used to detect edges, and then count the number of edge pixel. If the noise image is enhanced by NSCT, and the noise is enlarged, then the number of edge pixel is relatively large. If the noise image is enhanced by NSCT, and the noise is suppressed, then the number of edge pixel is relatively low. Usually the images have less noise, then the value of the image SNR is larger, and vice versa. If the parameter $m l$ is relatively large, then the weak edges and noise are suppressed. But the image SNR value is relatively large and the $\mathrm{DV} / \mathrm{BV}$ value is relatively large.

After testing many times, we take into account the characteristics of the details and noise, and the image enhancement evaluation function is proposed in this paper, as shown in equation (7). The evaluation function takes account of the definition of the image, image edges and the image noise. The more the Fitness is, the clearer the image is and the less noise the image has.

$$
\text { Fitness }=\frac{D v \times S n r}{B v \times E d g e_{N u m}}
$$

Where, $D v$ is detail variance. $B v$ is background variance. $S n r$ is the Signal to Noise Ratio of the enhanced image. $E d g e_{\text {Num }}$ is the number of edge pixel of the enhanced image.

In order to verify the effectiveness of the proposed image enhancement evaluation method, we compare the proposed method with the common image enhancement evaluation methods.

Figure 4(2)-(6) shows the enhanced images using equation 6 with different parameters in NSCT domain. Figure 4(1) is the original image. Through the subjective analysis, Figure 4(5) is the best image in the 6 images. Figure 4(2) and Figure 4(3) lose a lot of edges. Figure 4(4) and Figure 4(6) amplify the noise.

Figure 5 is the evaluation value of four methods to Figure 4 . The four methods are used to evaluate the enhanced images. The four methods are Brenner function [20], Wavelet transform [20], structural similarity index [21] (SSIM) and our method respectively. Our method is shown as equation 7.The Brenner function is shown as equation 8. The Wavelet method is shown as equation 9.

$$
F=\sum_{M} \sum_{N}(f(x+2, y)-f(x, y))^{2}
$$




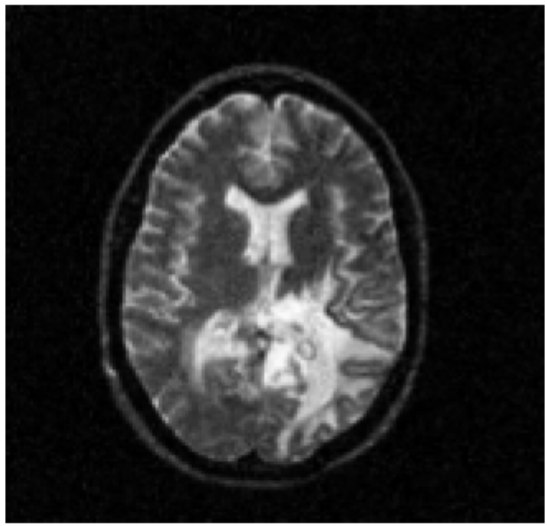

(1)

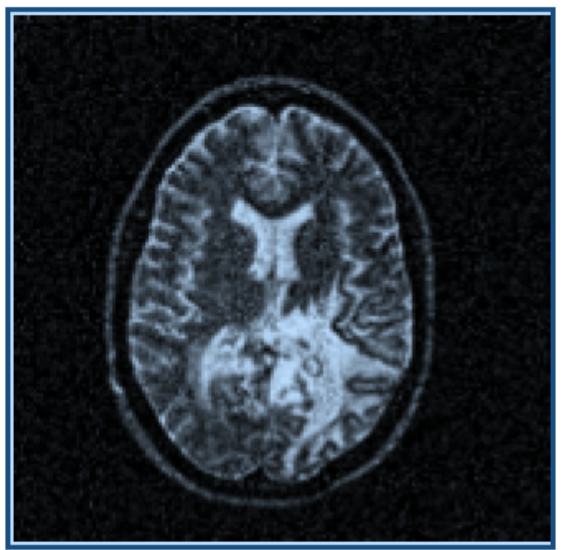

(4)

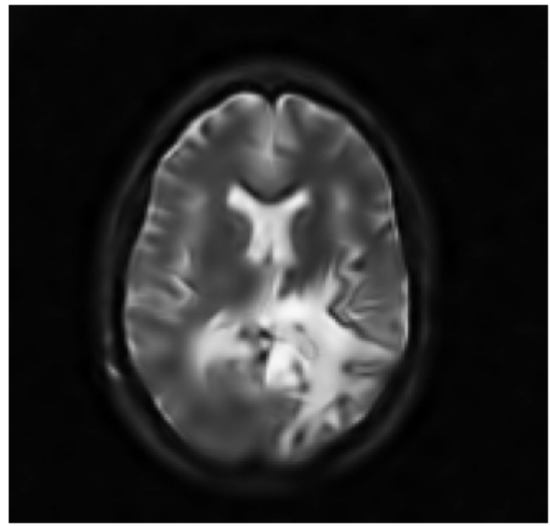

(2)

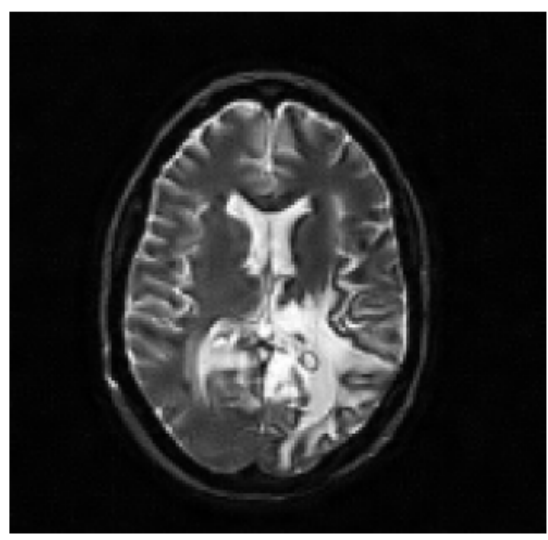

(5)

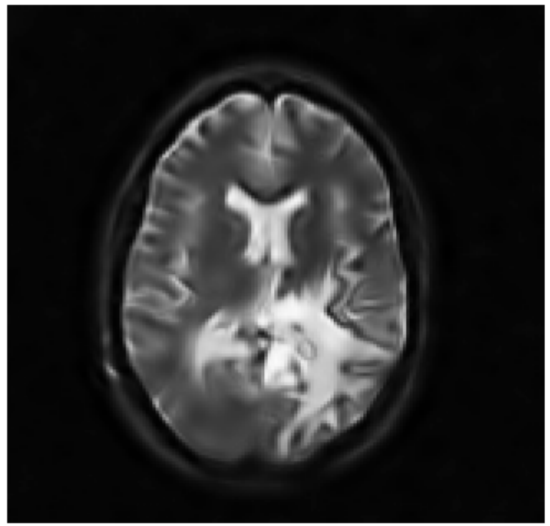

(3)

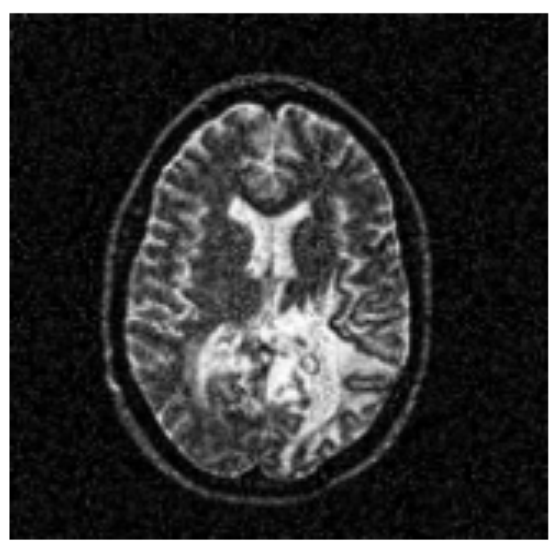

(6)

Figure 4. Image sequences

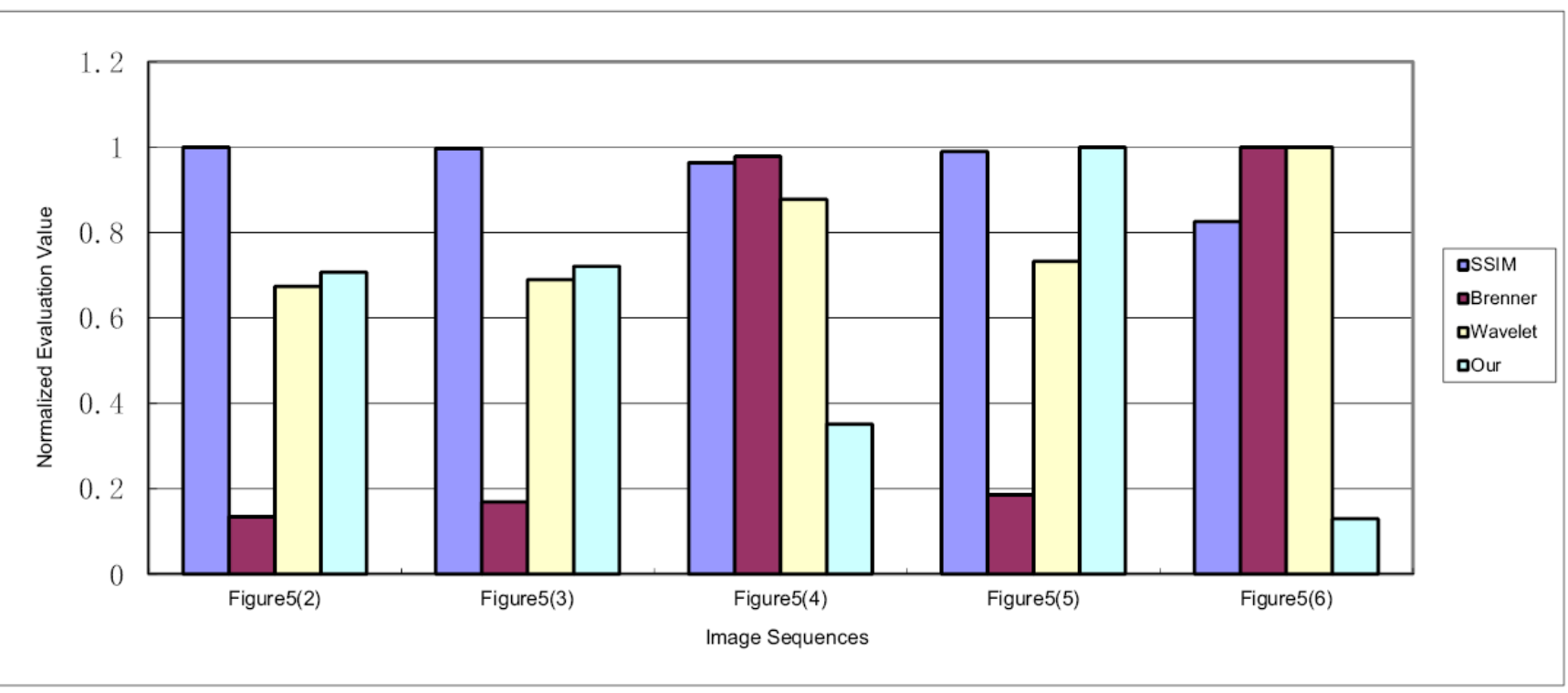

Figure 5. The evaluation value of four methods 
Where, $f(x+2, y)-f(x+2, y))^{2}>T h, T h$ is a certain threshold. The $f(x, y)$ represents a pixel value in the image. The $M$ and $N$ represents the size of the image.

$$
F=\sum_{i=1}^{N} \sum_{j=1}^{M}\left|W_{H L}(i, j)\right|+\left|W_{L H}(i, j)\right|+\left|W_{H H}(i, j)\right|
$$

Where, $W_{H L}(i, j), W_{L H}(i, j)$ and $W_{H H}(i, j)$ are the high frequency information of an image and represents respectively horizontal, vertical, and diagonal direction.

From Figure 5, we can know that our method gives the highest evaluation value to the Figure 4(5). Our method can evaluate the enhanced images accurately.

\subsection{Optimize Parameters by GA and Image Enhancement Algorithm}

In the field of artificial intelligence, a genetic algorithm (GA) [22] is a search heuristic that mimics the process of natural selection. This heuristic is usually used to generate useful solutions to optimization and search problems. The GA generates solutions to optimization problems using techniques inspired by natural evolution, such as inheritance, mutation, selection, and crossover. The evolution usually starts from a population of randomly generated individuals. More fit individuals are stochastically selected from the current population, and each individual's genome is modified to form a new generation. The selection operation is done based on individual fitness. The crossover operation is that two or more individuals exchange their genes to produce the next generation individual.

In this paper, the GA is adopted to optimize parameters. The parameters are $a, b, m 1, m 2$ respectively. The optimization procedure is as follows. The crossover probability is 0.8 , and the mutation probability is 0.05 . Initial population number is 20 . The maximum number of generation is 50 . Parameter optimization by GA is summarized in table 1 .

Input: The random four parameters: $a, b, m 1, m 2$

Step 1. Population initialization: set the evolution generation $t=0$, set the maximum evolution generation $T$, generate the $N$ individuals as the initial population $P(0)$ randomly. Every individual contains four parameters $a, b, m 1, m 2$. Shrink and stretch the NSCT coefficients using image enhancement function.

Step 2. Individual evaluation: compute the fitness of each individual according to the equation 7.

Step 3. Select operation: select the existing population to bear a new generation based on the individual fitness.

Step 4. Crossover operation: exchange the genes between two individuals based on crossover probability.

Step 5. Mutation operation: change the individual gene based on mutation probability.

Step 6. The next generation $P(t+1)$ was got through Select operation, crossover operation, mutation operation. Then the four parameters are optimized.

Step 7. If $t=T$, evolution to end

Output: Optimized four parameters: $a, b, m 1, m 2$.

Table 1. Parameters Optimization by GA

In this paper, we take the image enhancement evaluation function as the fitness function of the GA. Once the genetic representation and the fitness function are defined, a GA proceeds to initialize a population of solutions and then to improve it through repetitive application of the mutation, crossover, inversion and selection operators. Our image enhancement method is shown in Figure 6. 


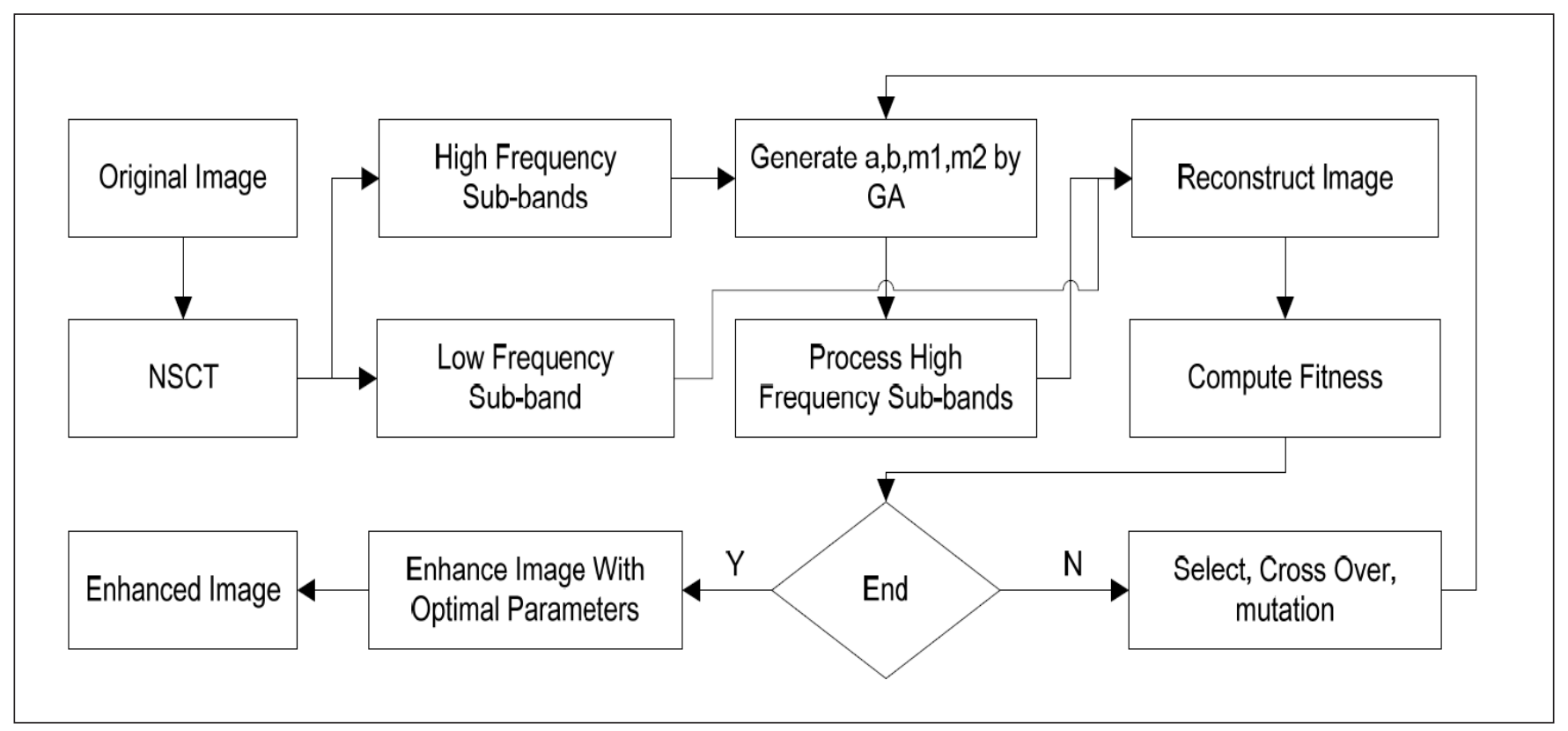

Figure 6. The proposed image enhancement method

\section{Experimental Results and Analysis}

In order to demonstrate the effectiveness of the proposed method in this paper, the Matlab is employed in numerical simulations. We use three standard images in our test suit: 'brain1', 'brain2' and 'Mandrill', which are given in figure.8. The three pictures are noised with Gaussian noise. The mean of noise is 0 and the variance of noise is 15 . And the experimental results are also compared with other methods, such as, bilateral filter, histogram equalization; guided filter and discrete wavelet transform based enhancement method. Experimental results are shown in Figure 8, Figure 9, and Figure 10. The discrete wavelet transform based enhancement method and the proposed method use the same enhancement function. The 'maxflat' pyramid decomposition and the 'pkva' directional filter bank are selected for NSCT decomposition. NSCT decomposition level is 3 and each level has eight high frequency directions. Discrete wavelet is selected. Wavelet base is ' $\mathrm{db} 1$ ' and wavelet decomposition level is 3. The PSNR of experimental results are shown in table 3 , table 5 , table 7 and the DV/BV of experimental results are shown in table 2 , table 4 and table 6.

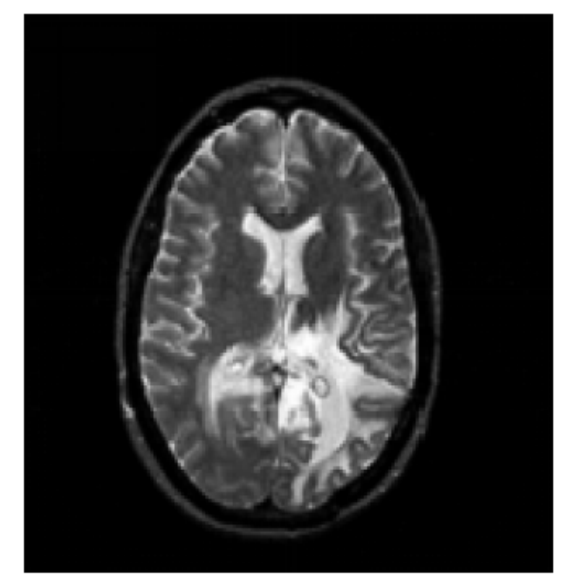

(1) Brain1

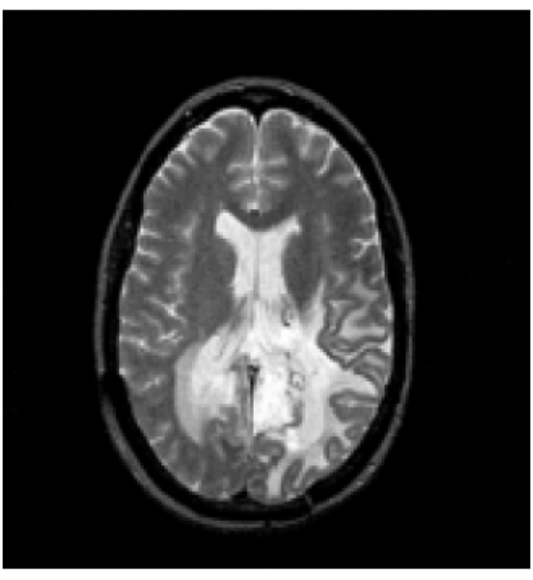

(2) Brain2

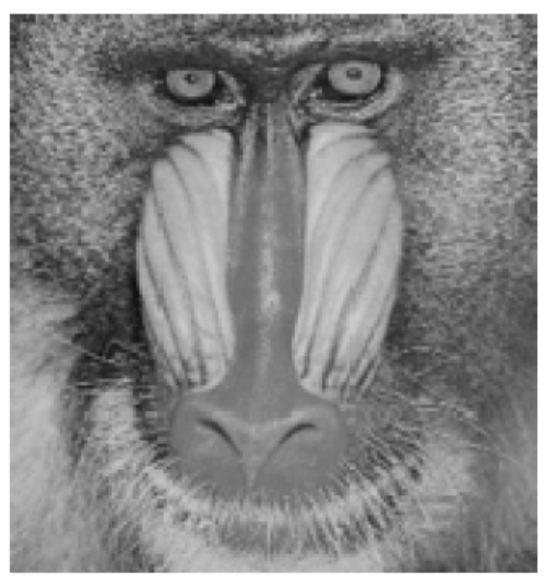

(3) Mandrill

Figure 7. Original images 
The brain 1 and the brain 2 are the magnetic resonance images of the human brain, and the mandrill is visible image.

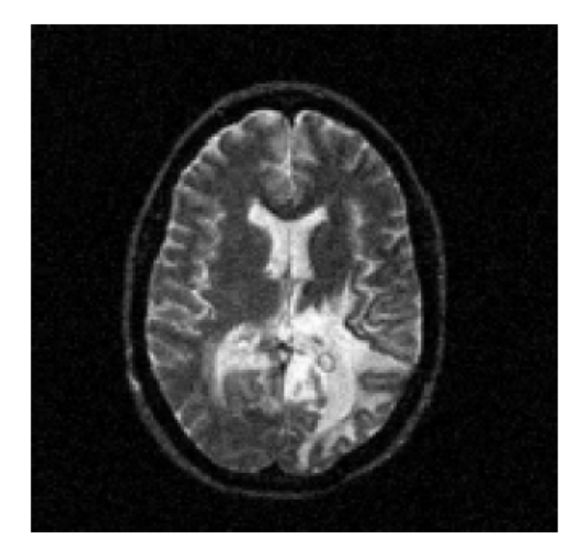

(1) Original image

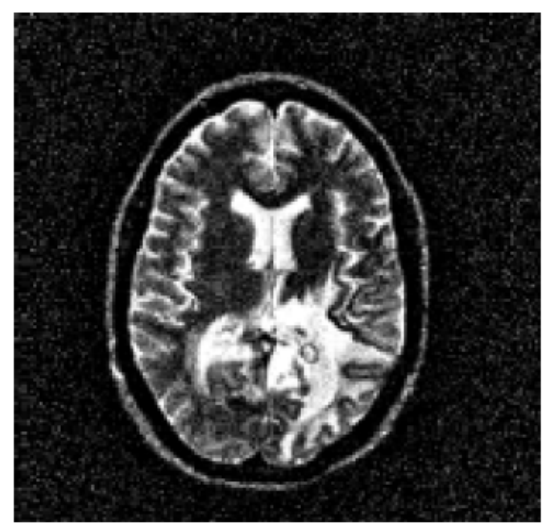

(4) Guided filter

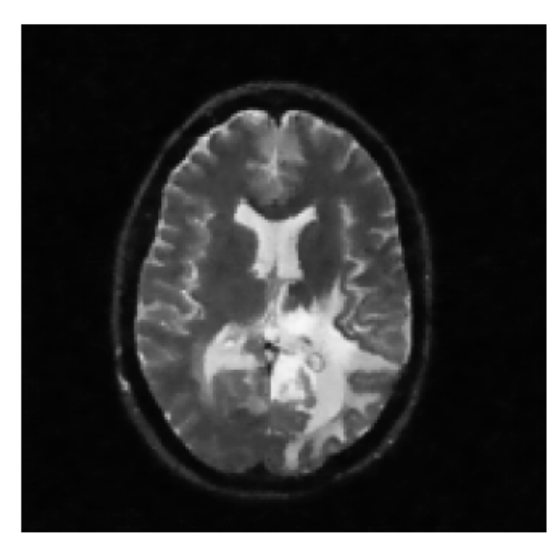

(2) Bilateral filter

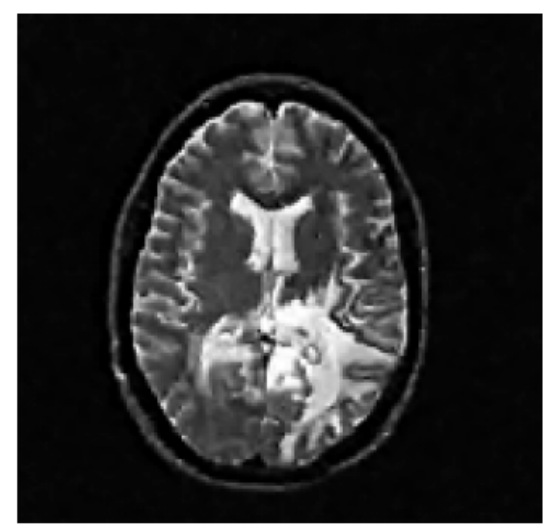

(5)Wavelet

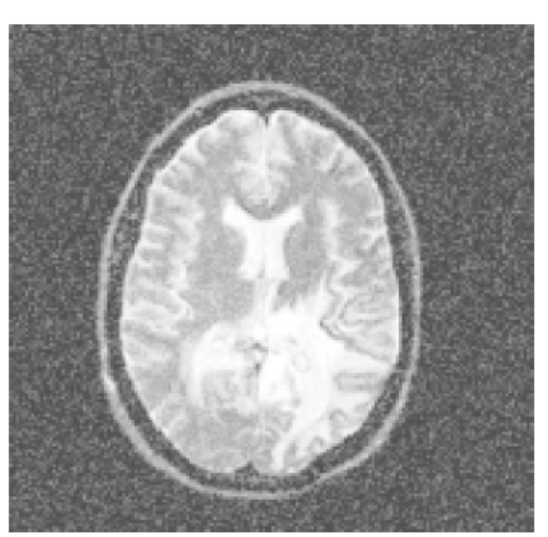

(3) HE

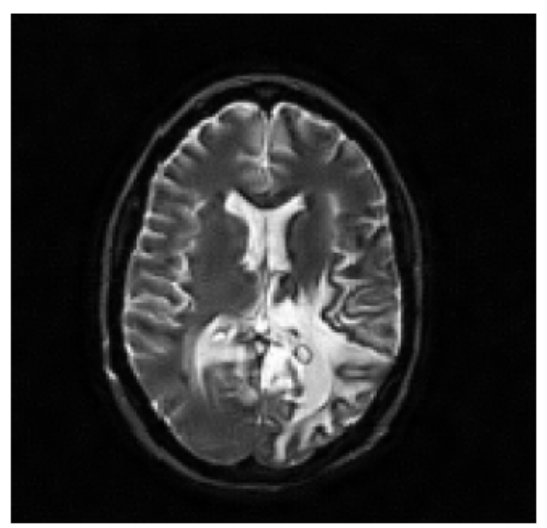

(6) Proposed method

Figure 8. Brain1 experimental results

\begin{tabular}{|l|l|l|l|}
\hline Brain1 & DV & BV & DV/BV \\
\hline HE & 26.28 & 5.94 & 4.42 \\
Bilateral filter & 23.55 & 2.06 & 11.42 \\
Guided filter & 33.11 & 4.54 & 7.29 \\
Wavelet & 24.14 & 1.58 & 15.19 \\
Proposed method & 28.27 & 1.79 & 15.70 \\
Noise image & 22.21 & 5.46 & 4.06 \\
Original image & 23.25 & 2.25 & 10.29 \\
\hline
\end{tabular}

Table 2. DV/BV of the enhanced brain1 


\begin{tabular}{|l|l|l|l|c|}
\hline HE & Bilateral filter & Guided filter & Wavelet & Proposed method \\
\hline 7.28 & 28.74 & 17.27 & 29.05 & 25.85 \\
\hline
\end{tabular}

Table 3. Peak signal-to-noise ratio of the enhanced brain1 (PSNR)

Figure 8 shows that the brain 1 image is enhanced by the bilateral filter method, the histogram equalization method, the guided filter method, the wavelet transform method and the proposed method respectively and the results are shown in Figure 8 (2) - (6) respectively. Figure 8 (1) is noised and we can see that the edges are blurred by the noise and it is difficult to identify image details. From the results we can see clearly that the bilateral filter method effectively suppresses the noise but blurs the edges at the same time. The histogram equalization method can effectively improve the contrast of the image, but the pixels with different gray levels are merged into the same gray level and the noise is amplified, then the details of the images are lost. The guided filter method can effectively enhance the image details. However, it is sensitive to noise, so the noise is amplified, and the details of the images are lost. The wavelet transform method can effectively suppress the noise while enhancing the image details. The proposed method, however, can significantly suppress the noise while enhancing the image details. And from Figure 8 (5) and Figure 8 (6), we can clearly see that the image details based on the proposed method are more obvious and smoother. The shapes of the skull are enhanced much better compared with other methods.

From table 2, we can clearly see that in the histogram equalization method and the guided filter method, the value of DV/BD falls, which means the image detail is blurred. In the bilateral filter method, the Wavelet transform method and the proposed method, all the values of DV/BV increase, all the values of DV increase and all the values of BD decrease. We can know that the three methods can effectively enhance the image details while suppressing the noise. However, the DV/BV value of the proposed method is the maximum value of the three methods which means the proposed method is better than other methods in this paper. From table.3, we can clearly see that the histogram equalization method and the guided filter can not suppress the noise. The bilateral filter method, the wavelet transform method and the proposed method can effectively suppress the noise.

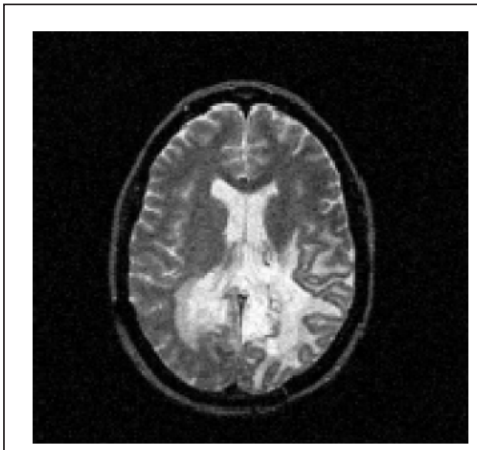

(1) Original image

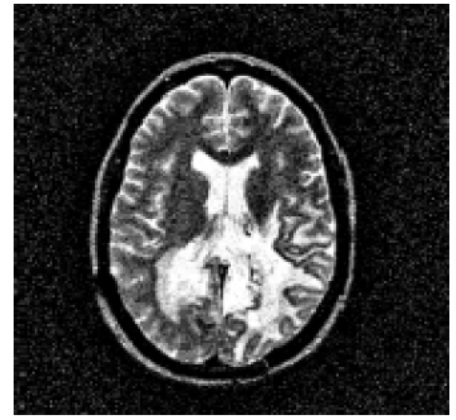

(4) Guided filter

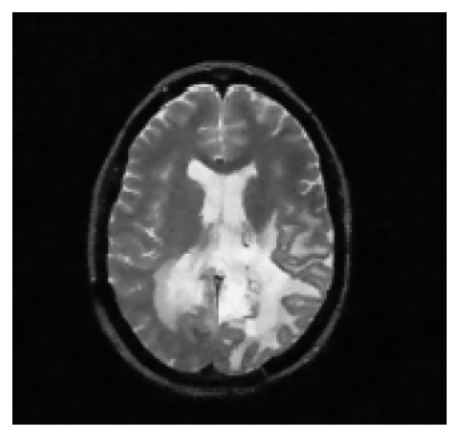

(2) Bilateral filter

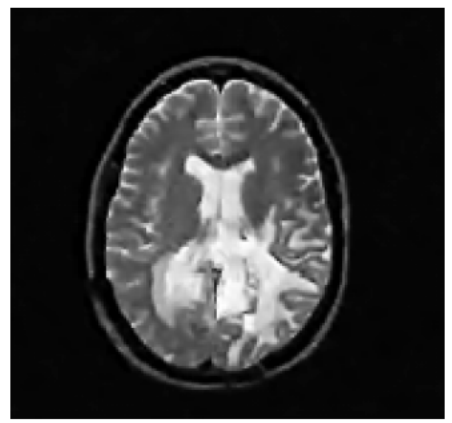

(5)Wavelet

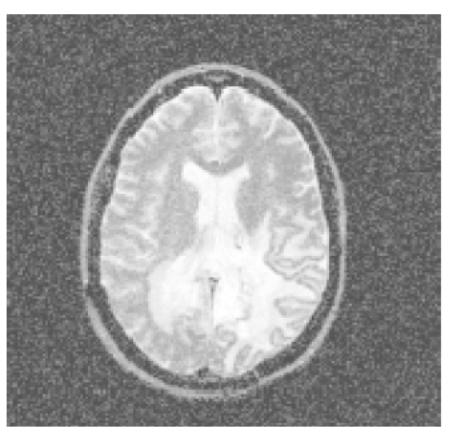

(3) HE

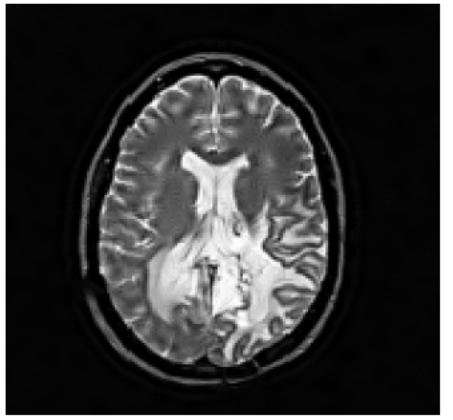

(6) Proposed method

Figure 9. Brain2 experimental results 


\begin{tabular}{|l|l|l|l|}
\hline Brain2 & DV & BV & DV/BV \\
\hline HE & 25.59 & 6.03 & 4.24 \\
\hline Bilateral filter & 26.05 & 1.99 & 13.04 \\
\hline Guided filter & 33.46 & 4.33 & 7.72 \\
\hline Wavelet & 31.73 & 1.56 & 20.33 \\
\hline Proposed method & 31.48 & 1.37 & 22.95 \\
\hline Noise image & 20.99 & 6.65 & 3.15 \\
\hline Original image & 25.20 & 1.50 & 16.74 \\
\hline
\end{tabular}

Table 4. DV/BV of the enhanced brain2

\begin{tabular}{|l|c|c|c|c|}
\hline HE & Bilateral filter & Guided filter & Wavelet & Proposed method \\
\hline 7.68 & 32.79 & 17.49 & 26.70 & 26.73 \\
\hline
\end{tabular}

Table 5. Peak signal-to-noise ratio of the enhanced brain2 (PSNR)

Figure 9 shows that the brain 2 image is enhanced by the bilateral filter method, the histogram equalization method, the guided filter method, the wavelet transform method and the proposed method respectively and the results are shown in Figure 9 (2) - (6) respectively. Figure 9 (1) is also noised and the edges are blurred. It shows that the bilateral filter can effectively suppress noise. But some edges are removed. The histogram equalization method improves the contrast at the cost of loss in image details. The guided filter method amplifies the noise while enhancing the edges. The wavelet transform method and the proposed method not only preserve the details but also improve the contrast of an image. However, the edges in Figure 9 (6) are clearer than these in Figure 9 (5). And the proposed method can get a better visual effect.

From table 4, we can see that both the value of $\mathrm{BD}$ and the value of DV/BD increase in the histogram equalization method and the guided filter method. So it means the two methods blur the image details. The value of the DV/BV in the bilateral filter method can effectively increase. It means that the bilateral filter method can effectively enhance the image details. The values of DV/BV significantly increase in the wavelet transform method and the proposed method. However, the proposed method can get a better value of DV/BV. So the proposed works are the best in enhancing the image details. From table 5, we can know that the proposed method can also do well in suppressing the noise.

Figure 10 shows that the Mandrill image is enhanced by the bilateral filter method, the histogram equalization method, the guided filter method, the wavelet transform and the proposed method respectively and the results are shown in Figure 10 (2) - (6) respectively. In Figure 10 (3), the histogram equalization can effectively improve the global contrast of the image. But the pixels with different gray levels are merged into the same gray level, then the details of the images are lost, such as the shape of the nose of gorilla in Figure 10 (3). Because the noise is amplified, the image processed by guided filter loses details and there are some false edges in the final images. The Bilateral filter works well in suppressing while enhancing the image details. The image noise can be effectively eliminated by the wavelet method. But the wavelet just has two directions, so it can not capture more directions. The NSCT can capture more contours in images, for example, the orangutans beard in Figure 10 (6) are more continuous than that in Figure $10(5)$.

In table 6 , the $\mathrm{DV} / \mathrm{BV}$ of the proposed method is the highest in all methods, which means that the image processed by the proposed method is the clearest. From table 7, the PSNR of the proposed method, the wavelet transform method and the bilateral filter is relatively high. It shows that the bilateral filter works well in de-noising. And it also shows that the proposed method and 


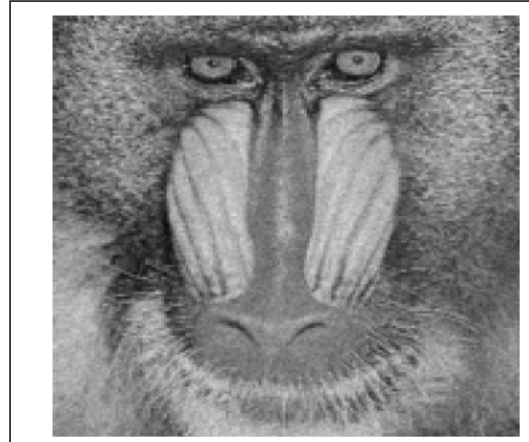

(1) Original image

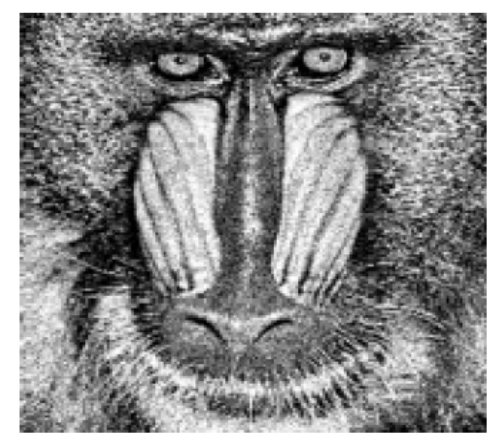

(4) Guided filter

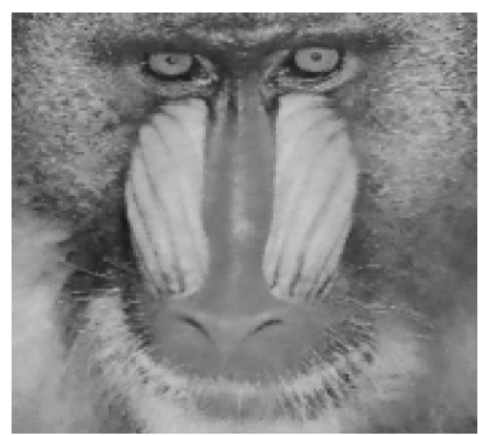

(2) Bilateral filter

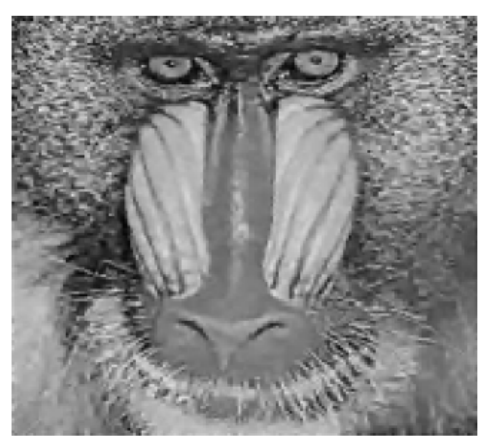

(5)Wavelet

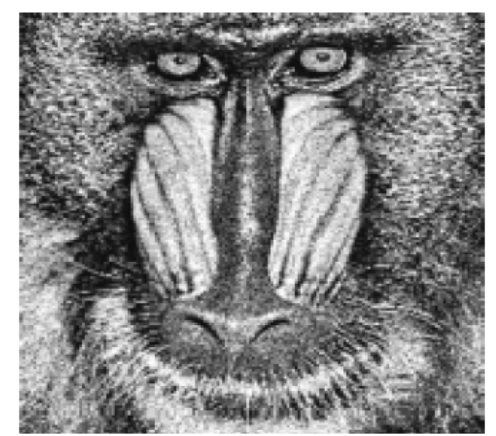

(3) $\mathrm{HE}$

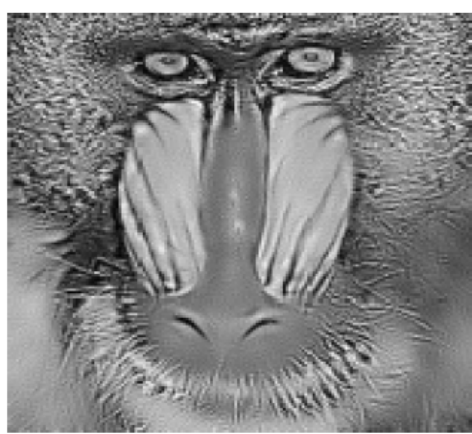

(6) Proposed method

Figure 10. Mandrill experimental results

\begin{tabular}{|l|l|l|l|}
\hline Mandrill & DV & BV & DV/BV \\
\hline HE & 50.21 & 9.75 & 8.72 \\
\hline Bilateral filter & 20.10 & 6.10 & 3.29 \\
\hline Guided filter & 51.59 & 7.12 & 7.24 \\
\hline Wavelet & 34.17 & 4.26 & 6.85 \\
\hline Proposed method & 40.02 & 3.83 & 10.44 \\
\hline Noise image & 23.19 & 8.61 & 2.69 \\
\hline Original image & 22.45 & 6.07 & 3.69 \\
\hline
\end{tabular}

Table 6. DV/BV of the enhanced Mandrill

\begin{tabular}{|l|c|c|c|c|}
\hline HE & Bilateral filter & Guided filter & Wavelet & Proposed method \\
\hline 14.61 & 27.54 & 14.91 & 22.17 & 23.30 \\
\hline
\end{tabular}

Table 7. Peak signal-to-noise ratio of the enhanced Mandrill (PSNR) 
the wavelet do well in de-noising.

\section{Conclusions}

Given all this, the proposed method in this paper adaptively suppresses the noise while enhancing the images details, which can overcome the shortcomings of traditional methods. The proposed can effectively enhance magnetic resonance images and visible images. We proposed an image enhancement function and an image evaluation criterion. The image enhancement function is used to nonlinearly shrink and stretch the NSCT coefficients. The proposed image evaluation criterion was used to evaluate the fitness of individuals. The optimal parameters of image enhancement function are chosen by genetic algorithm. And it outperforms Wavelet based on the same enhancement function with fixed parameters. Because four parameters need to be optimized, this method runs slowly. How to reduce the time complexity of the algorithm becomes the next research emphasis.

\section{References}

[1] Gonzalez, R C., Woods, R E., Masters, B R. (2009). "Digital image processing, third edition," Journal of Biomedical Optics, Vol. 14, No.6, pp. 029901, 2009.

[2] Ling, Z., Liang, Y., Wang, Y., et al. (2015). “Adaptive extended piecewise histogram equalization for dark image enhancement,” IET Image Processing, Vol. 9, No. 11, pp. 1012-1019, 2015.

[3] Liu, N., Zhao, D. (2014). "Detail enhancement for high-dynamic-range infrared images based on guided image filter," Infrared Physics \& Technology, Vol. 67, pp. 138-147, 2014.

[4] Kanwal, N., Girdhar, A., Gupta, S. (2011). "Region Based Adaptive Contrast Enhancement of Medical X-Ray Images," Bioinformatics and Biomedical Engineering, (iCBBE) $20115^{\text {th }}$ International Conference on. IEEE, pp. 1-5, 2011.

[5] Singh, K., Kapoor, R. (2014). “Image enhancement using Exposure based Sub Image Histogram Equalization,” Pattern Recognition Letters, Vol1. 36, No. 1, pp. 10-14, 2014.

[6] Liu, N., Zhang, Y., Xie, J. (2015). “A Novel High Dynamic Range Image Enhancement Algorithm Based on Guided Image Filter,” Optik - International Journal for Light and Electron Optics, Vol. 126, No. 23, pp. 4581-4585, 2015.

[7] Di, H., Gao, D. (2014). "Gray-level transformation and Canny edge detection for 3D seismic discontinuity enhancement," Computers \& Geosciences, Vol. 72, pp. 192-200, 2014.

[8] Jin, L., Xiong, C., Liu, H. (2012). "Improved bilateral filter for suppressing mixed noise in color images," Digital Signal Processing, Vol. 22, No. 6, pp.903-912, 2012.

[9] Cho, D., Bui, T D. (2014). “Fast image enhancement in compressed wavelet domain,” Signal Processing, pp. 295-307, 2014.

[10] Shi, J., Shan, Z. (2012). “Image resolution enhancement using statistical estimation in wavelet domain,” Biomedical Signal Processing \& Control, Vol. 17, No.6, pp. 571-578, 2012.

[11] Metwalli, M R., Nasr, A H., Faragallah, O S. (2014). "Efficient pan-sharpening of satellite images with the contourlet transform," International Journal of Remote Sensing, Vol. 35, No. 5, pp. 1979-2002, 2014.

[12] Xia, C., Jiao, L., Liu, F. (2015). "SAR Image Despeckling Using Scale Mixtures of Gaussians in the Nonsubsampled Contourlet Domain," Chinese Journal of Electronics, Vol. 24, No. 1,pp: 205-211, 2015.

[13] Changdong, Wu., Zhigang, Liu., Hua Jiang. (2014). "Catenary image enhancement using wavelet-based contourlet transform with cycle translation,” Optik, Vol. 125, No. 15, pp. 3922-3925, 2014.

[14] Shen, Y., Ren, E., Dang, J W. (2013). “A Nonsubsampled Contourlet Transform Based Medical Image Fusion Method,” Information Technology Journal, Vol. 12, No. 4, 2013.

[15] Wu, C., Liu, Z., Jiang, H. (2014). “Catenary image enhancement using wavelet-based contourlet transform with cycle translation,” Optik - International Journal for Light and Electron Optics, Vol. 125, No. 15, pp. 3922-3925, 2014.

[16] Bamberger, R H., Smith, M J T. (1992). A filter bank for the directional decomposition of images: theory and design[J]. IEEE Transactions on Signal Processing, 1992, 40 (4), 882-893. 
[17] Starck, J L., Murtagh, F., Candès, E J., et al. (2003). Gray and color image contrast enhancement by the curvelet transform, IEEE Transactions on Image Processing A Publication of the IEEE Signal Processing Society, 2003, 12(6) 706-17.

[18] Sezan, M I.,Tekalp, A M., Schaetzing, R. Automatic anatomically selective image enhancement in digital chest radiography, IEEE Transactions on Medical Imaging, 8 (2) 154-162, 1989.

[19] S karabot A., Ram poni G., T offoli D. (200). Image sequence processing for videowall visualization, In: Proceedings of SPIE ,Vol. 3961, p. 138-147,2000.

[20] Zhang, X., Wu, H., Ma, Y. (2015). A new auto-focus measure based on medium frequency discrete cosine transform filtering and discrete cosine transform, Applied \& Computational Harmonic Analysis, 2015, 40 (2) 430-437.

[21] Wang, Z., Bovik, A C. (2009). Mean squared error: Love it or leave it? A new look at Signal Fidelity Measures, IEEE Signal Processing Magazine, 2009, 26 (1) 98-117.

[22] Elsayed, S M., Sarker, R A., Essam, D L. (2014). A new genetic algorithm for solving optimization problems," Engineering Applications of Artificial Intelligence, 27 (1) 57-69, 2014. 